\title{
Performance Analysis of OLSR Protocol in MANET CONSIDERING DIFFERENT MOBILITY SPEED AND NETWORK DENSITY
}

\author{
Koay Yong Cett, Nor Aida Mahiddin*, Fatin Fazain Mohd Affandi, \\ Raja Hasyifah Raja Bongsu, Aznida Hayati \\ Faculty of Informatics and Computing, \\ Universiti Sultan Zainal Abidin (UniSZA) Terengganu, Malaysia
}

\begin{abstract}
A Mobile Ad Hoc Network (MANET) is created when an independent mobile node network is connected dynamically via wireless links. MANET is a self-organizing network that does not rely on pre-existing infrastructure such as wired or wireless network routers. Mobile nodes in this network move randomly, thus, the topology is always changing. Routing protocols in MANET are critical in ensuring dependable and consistent connectivity between the mobile nodes. They conclude logically based on the interaction between mobile nodes in MANET routing and encourage them to choose the optimum path between source and destination. Routing protocols are classified as proactive, reactive, or hybrid. The focus of this project will be on Optimized Link State Routing (OLSR) protocol, a proactive routing technique. OLSR is known as the optimized variant of link state routing in which packets are sent throughout the network using the multipoint relay (MPR) mechanism. This article evaluates the performance of the OLSR routing protocol under condition of changing mobility speed and network density. The study's performance indicators are average packet throughput, packet delivery ratio (PDR), and average packet latency. Network Simulator 2 (NS-2) and an external patch UM-OLSR are used to simulate and evaluate the performance of such protocol. As a result of research, the approach of implementing the MPR mechanism are able to minimise redundant data transmission during the normal message broadcast. The MPRs enhance the link state protocols' traditional diffusion mechanism by selecting the right MPRs. Hence, the number of undesired broadcasts can be reduced and limited. Further research will focus on different scenario and environment using different mobility model.
\end{abstract}

\section{KEYWORDS}

MANET, OLSR, Node Mobility, Density, Routing Scheme

\section{INTRODUCTION}

MANET is a wireless network that gaining a great attention due to being self-organizes, selfconfigures and self-repairing [1]. It is easily created by an autonomous system of wirelessly connected mobile nodes and can be deployed even without assistance of external infrastructure, such as a centralised base station (BS) or access point (AP). It is a fixed in the short that may be set up at any moment and in any location as a reserve in the scenario where physical infrastructure gets insufficient. For instance, MANET was chosen as the primary network to be used during disaster area where existing and physical infrastructure may have been destroyed, resulting in a massive breakdown in communication. Historical evidence demonstrates that the communication demand increases dramatically, which later leads to a major occurrence. The improvement in the wireless communication plays a crucial role especially in emergency and search and rescue (SAR) operations due to improvement in end user hardware such as mobile 
International Journal of Wireless \& Mobile Networks (IJWMN), Vol.13, No.6, December 2021

devices and other electronic devices [1]. To accommodate a variety of SAR operations during the disaster area scenario, the MANET may be efficiently altered, minimizing loss of life and property [2]. Due to the fact that MANET is a self-configuring, self-repairing, and selfrecovering network, it is easy to cope even in the absence of the established infrastructure [3], [4]. The communication network may be resolved quickly, minimizing delays and errors during the SAR operations. Thus, the MANET can be regarded as a pragmatic solution for conducting the SAR operations during disaster area because of its quick deployment, which enables rescue teams to react quickly to victims' calls [5], [6].

MANETs consist of multiple nodes particularly electronic devices such as tablets, smartphones, and video cameras. All of this equipment is connected via the wireless network and may communicate with each other. All mobile nodes are free to join or leave the network at any moment. The mobile nodes can move freely and be self-organized. As a result, the network's topology might undergo rapid and unpredictable changes. Additionally, each of the network nodes can act as a recipient, transmitter, or intermediate node that acts as a router, which transmitting data to and from other mobile nodes. In the real-world scenarios, the nodes are very mobile and relies on batteries to operate, considering the MANET applications [7].

Routing in MANET is based on a simple approach that allows each of the nodes to re-emit the data message for the ease of propagation across the network from a source to its precise destination. The critical issue in routing protocol design is determining their optimal path to the destination. These protocols assist the nodes in determining the ideal path for packets to go through the network. It is also used to develop and maintain an updated routing information that enables the nodes to determine their ideal route for the communication between the source and destination node. A few routing techniques have been proposed to address the issues with high mobile nodes where topology changed frequently [8].

The paper is organized as follows: Section 2 explains the classification of routing protocols. In Section 3, explains related works involving routing protocols in measuring the network performance of MANET. Section 4 describes proposed approach in routing mechanism considering OLSR routing protocol. Section 5 describes the network parameters used for the simulation. Section 6 presents the results and analysis of the proposed approach. Finally, conclusion is drawn in Section 7.

\section{Classification of Routing Protocols}

Ad hoc routing protocol is a commonly used protocol referring to the protocol that aid in determining the paths to be used for routing packets between the source and destination of the nodes in the MANET environment. The role of routing protocols is to maintain routing tables containing all available routing information and preserve a router's table dynamically [1]. In the ad hoc networks, the nodes had limited information regarding their networks' topology. Thus, the routing can be quite challenging in MANETs due to the restricted resources and random movement of nodes. As a result, a routing algorithm is presented to resolve the dilemma and determine the ideal path between the origin and destination nodes.

\subsection{Proactive Protocol}

Furthermore, it is a table-driven protocol that makes use of mapping tables to preserve the route and course of each node within the defined network. This provides the routing scheme by continuously sending topological knowledge packets of data to the network's nodes. As a consequence, each node's routing information is regularly updated leveraging the updated 
International Journal of Wireless \& Mobile Networks (IJWMN), Vol.13, No.6, December 2021

topological information. When nodes are added or removed from the network, control messages are sent to neighbouring nodes and routing tables are modified. Typically, this network protocol makes extensive use of link state techniques to disseminate information about its network neighbours [9].

\subsection{Reactive Protocol}

On-demand protocols are so named because they initiate a path discovery procedure once the source node has network packets to disseminate to the destination node. Therefore, determining the shortest path between the communication nodes. Once the path is created, it will be retained until the pathway is no longer permitted or until the packets of information reach the target. A series of activities, including the usage of a sequence number, have been taken to retain the new route and avoid repetition.

\subsection{Hybrid Protocol}

It is a hybrid of proactive and reactive protocol techniques in which the strengths of both prior protocols are merged to create an immediate reactive neighbourhood linked to proactive connections up to a particular distance. If an application wishes to transfer packets to a node apart from this zone, a reactive evaluation is performed. This instantly makes the pathways within a node's coverage area available. Initially, proactive routing protocols are implemented using routing tables in association with root nodes. If the node learns that it lacks knowledge on the path to the destination of the source nodes, it will perform route discovery using the reactive routing scheme [10].

\section{RELATED WORKS}

Muthana Najim Abdulleh and Salman Yussof conduct a comparative evaluation of the routing protocol, considering the specific number of nodes and size of simulation area [11]. Throughput, average end-to-end (E2E) delay, packet delivery fraction (PDF), and normalized routing load (NRL) were used to evaluate the performance of the routing protocols. The simulation is carried out for two scenarios, with differences in simulation parameters studied between the two scenarios. The range number of the nodes in the first scenario was limited between 30 until 150 . In second scenario, they focus to the network density and scale the map from 500 to 1750 metres. The simulation results indicate that Greedy Parameter Stateless Routing (GPSR) outperforms OLSR and Ad hoc On-Demand Distance Vector (AODV) routing protocol in the majority of tests. Moreover, the simulation results indicate that increasing in terms of number of the nodes has a substantial effect on the NRL, whereas changing the map area of the simulated region has a considerable effect on throughput, average E2E delay, and PDF.

K.Natarajan and G.Mahadevan [12] perform an analysis on how the mobility speed can affect the performance of network routing protocols. The selected routing protocols are AODV, Destination Sequenced Distance Vector (DSDV), Dynamic Source Routing (DSR), Location-Aided Routing (LAR), OLSR, Fisheye State Routing (FSR) and Zone Routing Protocol (ZRP). The network stimulation is divided into three distinct scenarios: low mobility, medium mobility, and high mobility. The simulation results indicate that LAR and AODV perform better than other protocols, reliably transmitting around 50\% to $60 \%$ of data packets independent of speed. Additionally, the DSR protocol exhibits a delay of 53\% and $95 \%$ greater than the AODV and DSDV procedures, respectively. 
International Journal of Wireless \& Mobile Networks (IJWMN), Vol.13, No.6, December 2021

Moreover, Lakshman Naik L, R. U. Khan, and R. B. Mishra [13] examined the effectiveness of different ad hoc routing protocols by varying the speed of nodes. The aim of the paper is to analyse the influence of the mobility speed of a node on various routing techniques. The performance analysis will be conducted on AODV, DSDR, and OLSR routing protocols. They simulate the network using three different node speeds with a fixed number of source/sink links of ten. As a result, OLSR protocol has a higher throughput than AODV and DSDV when the node speed varies. Although OLSR performance degrades slightly as the node speed increases, it is still superior to AODV and DSDV. Besides, OLSR has a greater packet delivery ratio than AODV and DSDV. OLSR, on the other hand, degrades slightly as the node speed increases. In terms of E2E delay, OLSR outperforms both AODV and DSDV. However, when the node speed increases, OLSR suffers a minor decrease. The packet loss findings indicate that the OLSR outperforms AODV and DSDV, but the performance drops considerably as the node speed increases. Finally, they concluded that OLSR outperforms AODV and DSDV across all the parameters they considered.

Gouri M. Patil, Ajay Kumar, and A. D. Shaligram [14] conducted a performance comparison of the different ad hoc routing protocols considering the network area size. The OLSR, AODV, DSR, Gathering-based Routing Protocol (GRP) and Temporally Ordered Routing Algorithm (TORA) have been examined. The simulation of several routing methods is carried out with three different sizes of the simulation areas. The findings indicate that the TORA is the optimal choice when the network load is taking into a concern. For a scalable simulation area up to 2000x2000 square metres, the DSR is the second-best option, followed by AODV, OLSR, and GRP. However, if the E2E is taking into account as the factor, the GRP performs better when the network area is up to $1000 \times 1000$ square metres. For $2000 \times 2000$ square metres, the OLSR is the preferred option. For the throughput, AODV achieves the highest throughput in three scenarios considering varying the network area sizes.

In [15], D. Kumar and S.C. Gupta investigate the influence of varying the transmission ranges, the node density, and the nodes speed on OLSR, DSR and ZRP. These three routing protocols correspond to three types of the routing protocols used in mobile ad hoc networks which are proactive, reactive, and hybrid routing protocols. The simulation is conducted using three different scenarios by varying node density, transmission range, and node speed. Based on the simulation conducted by the authors, it showed that DSR outperforms OLSR and ZRP in terms of E2E delay and PDR.

Ashutosh Sharma and Rajiv Kumar [16] conducted a performance assessment of a number of different ad hoc routing methods used in MANET. Different performance indicators such as average throughput, average PDR, and average delay were used to compare different routing protocols. The AODV surpasses the other routing protocols in terms of average throughput. Additionally, the OLSR works optimally in the scenario of average PDR due to OLSR's acyclic path route selection. Besides, TORA is successful in dense networks since it broadcasts the information to all the nodes. Finally, DSR delivers the least network delay. It is concluded that the reactive protocols have a low average delay and a high throughput.

Authors in [17] proposed multi-metric performance of modified OLSR in which they implemented an energy-aware and link stability approach to the MPR selection. The proposed approach allowed a longer network lifetime by including the residual energy in the MPR selection criteria via willingness variable. The simulation is simulation via Network Simulation 3 (NS-3) under different scenarios, varying the number of nodes and mobility pattern. The simulation results showed a significant loss in terms of End-to-End (E2E) delay for the modified OLSR. 
International Journal of Wireless \& Mobile Networks (IJWMN), Vol.13, No.6, December 2021

According to R. H. R. Bongsu, A. Mohammed, and M. A. Mohamed in [18], Wireless Mesh Networks (WMNs) have garnered considerable attention and have been thoroughly researched in a variety of domains such as architecture, implementation, and protocol development. Numerous organisations have recognised the value of multi-radio multi-channel (MRMC), particularly in rural networks, battlefields, and natural disasters, where a rapid communication network is required for deployment. The WMN is made up of mesh nodes that are connected by gateways, routers, and clients.

Finally, Ako Muhammad Abdullah, Emre Ozen, and Husnu Bayramoglu [19] examined a variety of mobility models, including Fast Car Model (FCM), the Slow Car Model (SCM), the RaceWalking Model (RWM), and the Human Walking Model (HWM). The authors developed these mobility models by varying node speeds in order to evaluate the performance of the AODV, OLSR, and GRP protocols. Different performance metrics are used to evaluate the network performance. They concluded from the simulation results that OLSR protocols outperform two other routing methods. It can be regarded that OLSR protocol is the most appropriate and effective network routing protocol, allowing for minimal delay and retransmission attempts, as well as increased data transfer performance from the source to the destination target. Meanwhile, in all simulation scenarios, AODV protocol outperformed OLSR and GRP in respect of data drop rate and network load. In comparison to the GRP protocol, the HWM model had a quite higher AODV network load. Also, the GRP protocol has a substantially lower media access delay and a data rate than AODV in all simulation scenarios. The findings show that this sort of operation is critical in deciding the protocol to fully exploit in the network. For example, the OLSR protocol is highly suited for giving real-time assistance.

\section{Optimized Link State Routing (OLSR) Protocol}

OLSR is the routing protocol's equivalent implementation for the classical link-state. In comparison to the distance vector routing protocol, these routing methods avoid routing loops and have no scalability issues [9]. Due to the flooding method used by conventional connection state routing protocols, the communication of topological information between the nodes generates a substantial quantity of traffic. It is an unfavourable characteristic of the MANET as a result of insufficient resources. The OLSR implements a new method in the network in order to minimize the traffic volume involved. All OLSR nodes are permitted to receive data packets including topological information, but only a small number of nodes referred to as MPRs are permitted to relay messages across the network. MPRs are the smallest number of neighbours that a node needs to communicate with all of its other neighbours within two hops [13].

The OLSR protocol is built around two main topology management mechanisms: neighbourhood detection and neighbourhood sensing. The OLSR protocol makes use of both these processes via four distinct types of control messages: HELLO, TC, MID, and HNA. Also, OLSR protocol does neighbourhood sensing via HELLO packets [2]. The topological data is transferred by using optimal dissemination or MPRs to scatter the TC packets. The TC messages are a collection of links in the neighbourhood of the network nodes that are used to handle the OLSR protocol's packets [20].

Further, OLSR protocol takes into account all interfaces associated to the network nodes when transmitting MID messages. As a result, the network's nodes can exploit all available routes regardless of the type of interfaces utilised at each hop. OLSR node assigns a primary address to one of its interfaces, which can later be used as a pointer in control messages. Likewise, the OLSR protocol's HNA messages are used to construct sub networks and hosts that exist outside of MANET but are discoverable by a node functioning as a gateway [7]. 
International Journal of Wireless \& Mobile Networks (IJWMN), Vol.13, No.6, December 2021

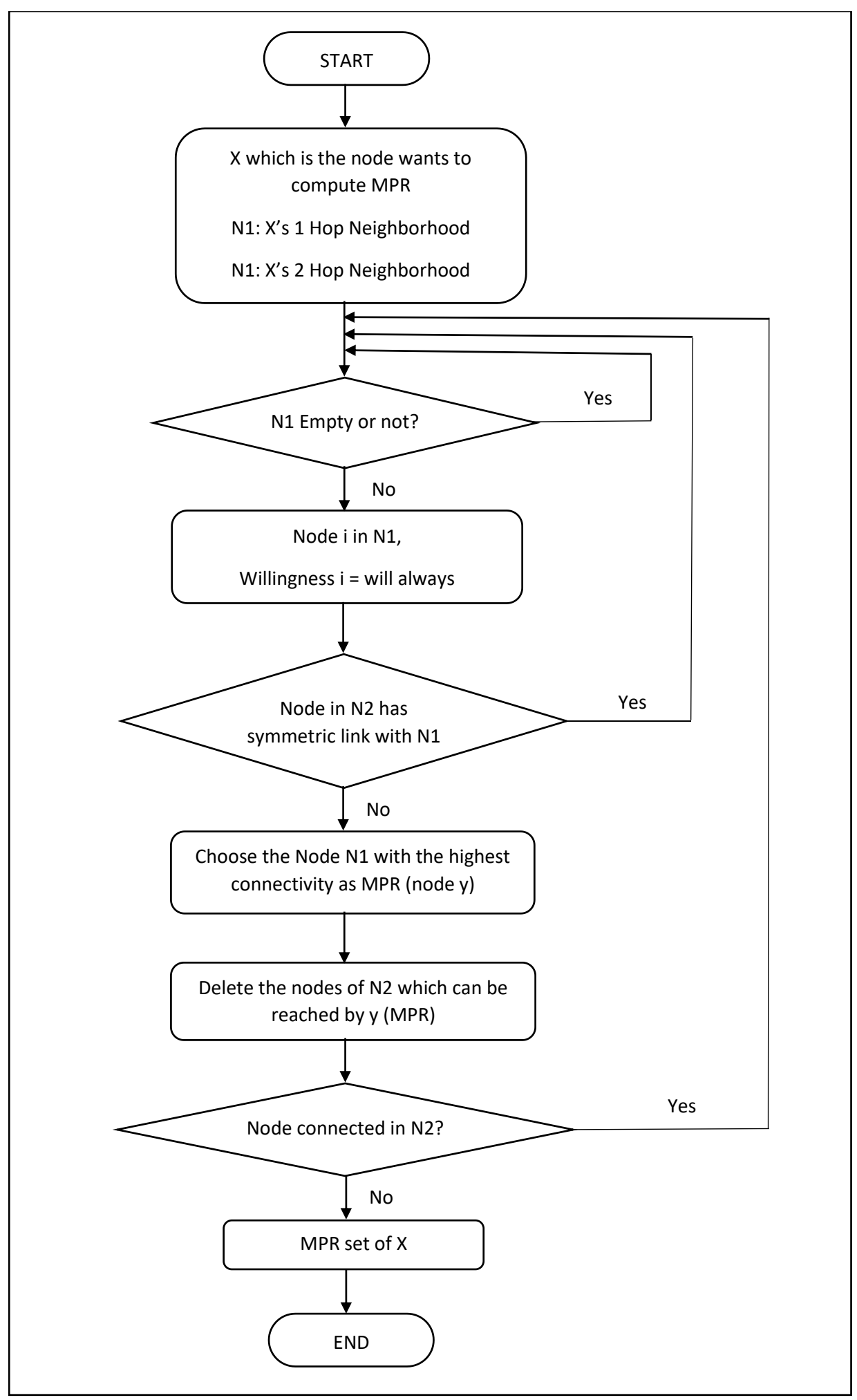

Figure 1. Routing selection technique

The fundamental goal of MPRs is to minimise redundant data transmission during the normal distribution of the message. MPRs are very helpful when it comes to delivering control 
International Journal of Wireless \& Mobile Networks (IJWMN), Vol.13, No.6, December 2021

information over a network. MPRs enhance the link state protocols' traditional diffusion mechanism. A given node chooses a group of MPRs depending on its information of its two-hop neighbourhood.

In a MANET, with a dynamically shifting topology, the MPRs had to be re-evaluated anytime the two-hop neighbour set's experience changed. As a result, the MPRs' position in the neighbourhood is restricted [18]. The improvement in packet diffusion methods employing the MPR methodology is illustrated in Figure 1.

In the first part of the figure, the central node distributes control information to eight additional nodes through the traditional flooding mechanism. The relay approach is illustrated in the following figure, with the message being carried via four nodes. Thus, by choosing the right MPRs, it is possible to limit the number of undesired broadcasts. By retaining a variety of data tables, OLSR protocol functions as a modification of the traditional link-state protocol in neighborhood sensing. Every time a control information is conveyed or broadcast, the tables are reset. The nodes save a variety of unique tables in the cache.

Moreover, the HELLO messages have three distinct functions in the OLSR protocol. For link sensing and neighbor sensing, the messages are conveyed to the neighbor within one hop, and for two-hop sensing, they are sent to the neighbor within two hops. Finally, it acts as an MPR selector sensor, indicating the presence of MPRs in the network [7].

Furthermore, the OLSR is referred to as a proactive and table-driven routing system. The routing loops do not exist in the link state routing protocols. Also, the link state routing techniques are scalable. However, the link state routing protocol creates a huge quantity of traffic when the topological data is sent between mobile nodes. Due to the restricted resources available in the MANET, excessive traffic is an unfavorable characteristic [20]. The OLSR protocol introduced a novel strategy or technique for significantly reducing the amount of bandwidth required to exchange the topological data between nodes. The OLSR protocol approves and permits all nodes to receive the topological data messages.

Nevertheless, only a few MPRs are capable of delivering all of this information throughout the network. To conclude, a node's MPRs are the fewest immediate neighbors needed to communicate all its neighbors in two hops. Thus, the MPRs ensure the information messages linked with the entire network reaches every node.

\section{Simulation}

The simulation of the project is performed by using NS-2. NS-2 is one of the commonly used network simulator for MANET and Vehicle Ad hoc Network (VANET). NS-2 supports both wired and wireless networks for routing and multicast protocols. In this study, NS-2 is chosen instead of other network simulator is due to its being used widely by other researchers and its simplicity in simulating the network. NS-2 is more practical to simulate the network than Network Simulator 3 (NS-3) as there are many references that study such protocol. Although NS3 is newest than NS-2, however there are limited studies and guidance in simulating the network. Hence, NS-2 is more practical to be used as the researchers can compare the results with other studies due to wide range of resources that were using the same simulator but, under different approaches and metrics. Using the same simulator as other studies can be one of our ways to ensure that the project being conducted in the right path.

Based on the summarization of the previous work, the simulation parameters for the project are proposed. The selected simulation area is $1000 \mathrm{~m}$ x $1000 \mathrm{~m}$. In addition, the number of the nodes 
International Journal of Wireless \& Mobile Networks (IJWMN), Vol.13, No.6, December 2021

selected in the following project are 20,40,60, 80 and 100 nodes respectively. The number of the nodes above 100 are not selected as the simulation requires too much time to compute in the current machine used. The mobility speeds are varied into 5 situations which are very slow mobility (VSM), slow mobility (SM), moderate fast mobility (MFM), fast mobility (FM) and very fast mobility (VFM). The VSM is stated as $10 \mathrm{~m} / \mathrm{s}$, SM is stated as $15 \mathrm{~m} / \mathrm{s}$, MFM is stated as $20 \mathrm{~m} / \mathrm{s}$ and FM is stated as $20 \mathrm{~m} / \mathrm{s}$. The simulation time limit is set to $900 \mathrm{~s}$ which is the maximum simulation time in the research papers. The parameters are summarized in Table 1 below.

Table 1. Simulation parameters.

\begin{tabular}{|l|l|}
\hline Parameter & Specification \\
\hline Simulation Area $(\mathrm{m} \mathrm{x} \mathrm{m)}$ & $1000 \times 1000$ \\
\hline Mobility Model & Random Waypoint \\
\hline Routing Protocol & OLSR \\
\hline Number of Nodes & $20,40,60,80,100$ \\
\hline Speed (m/s) & $10,15,20,25,30$ \\
\hline Simulation Time Limit (s) & 900 \\
\hline Mac Type & 802.11 \\
\hline Traffic & CBR \\
\hline Packet Size (bytes) & 512 \\
\hline
\end{tabular}

\section{RESUltS}

The considered performance metrics of this project are average delay, average throughput and PDR to evaluate the network performance of the proposed routing technique.

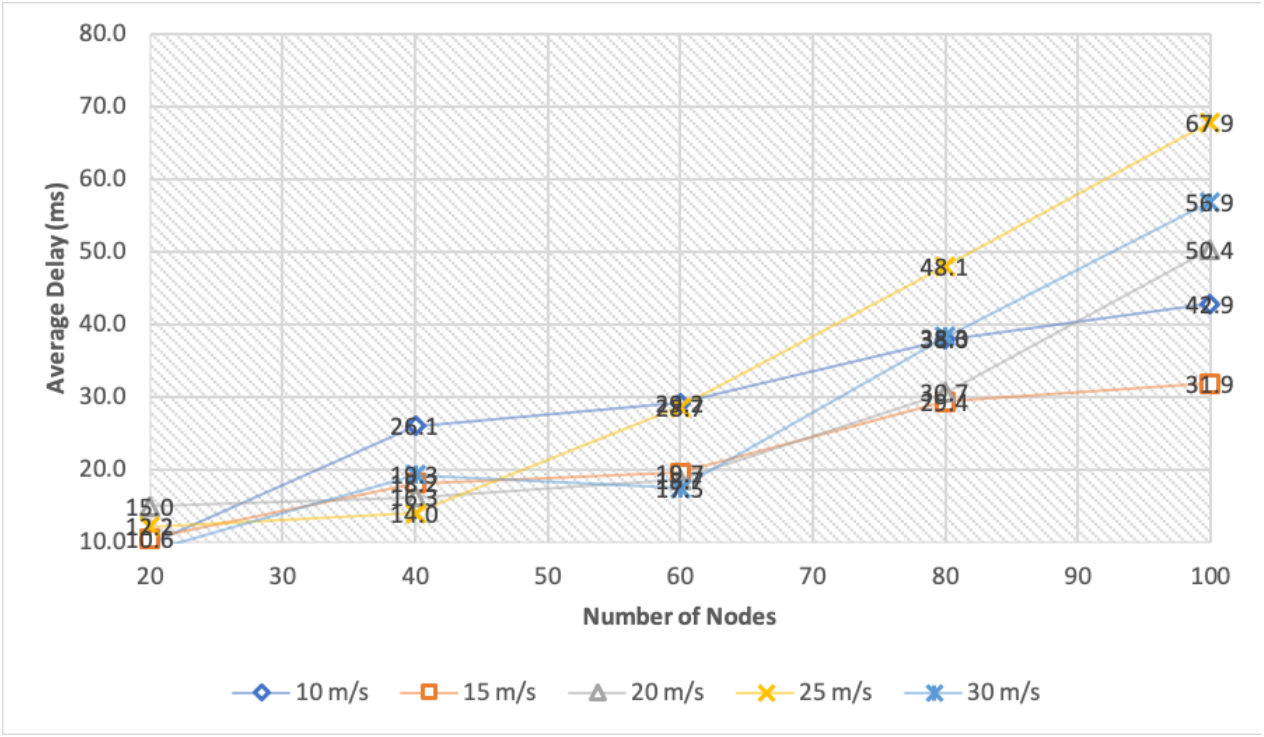

Figure 2. Average delay

As shown in Figure 2, the increasing in delay is due to the increase in number of nodes. FM illustrates that average delay increased in a gradual speed as the number of nodes increased. In addition, FM has the highest average delay when it reaches 100 nodes while, VFM has the lowest average delay when the number of nodes is set at 20 in the simulation. Besides, the average delay for 100 nodes in the simulation is the highest in all various mobility speeds. Therefore, the average delay is the lowest when the number of nodes is less. 


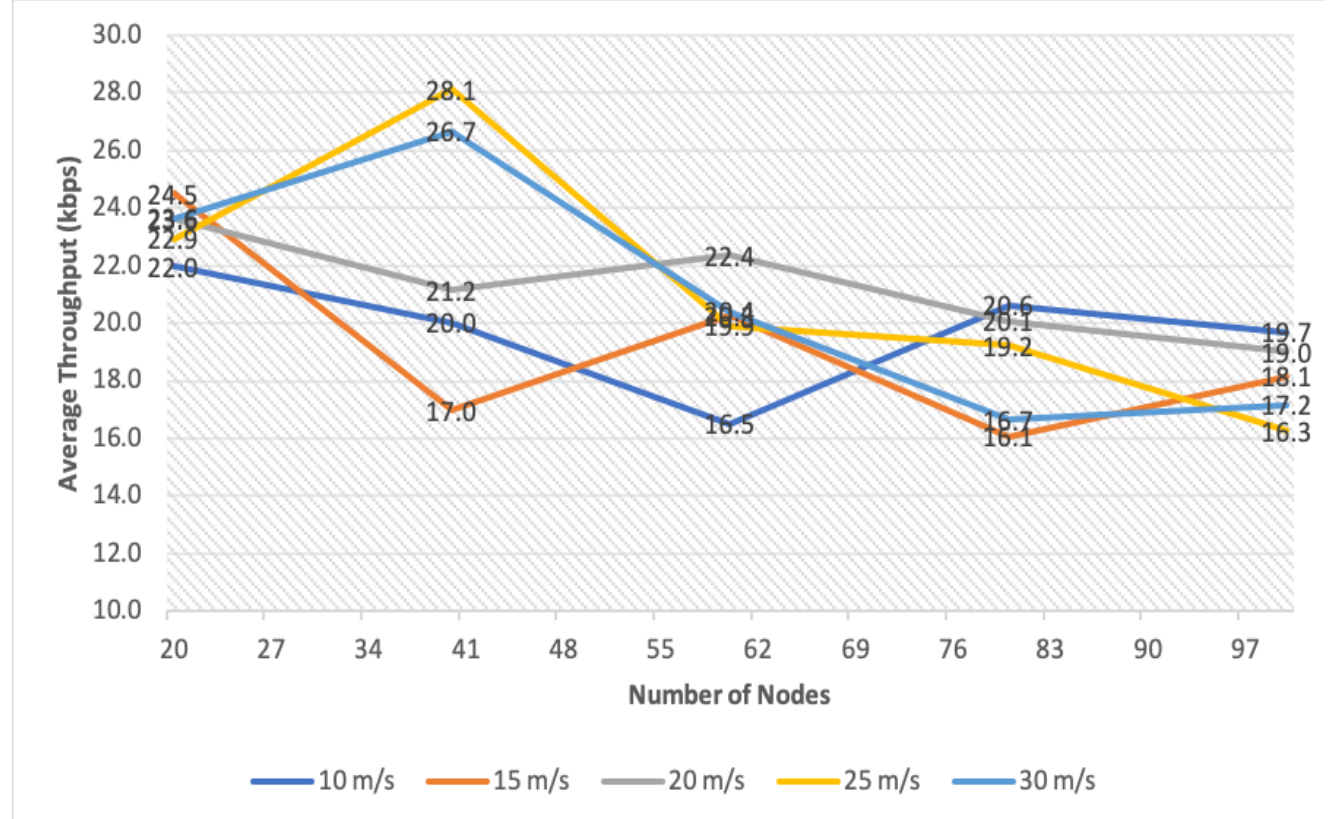

Figure 3. Average throughput.

Figure 3 illustrated the results of throughput obtained in the network simulation. From the results, we notice that FM that is associated with 40 nodes in the simulation has the highest average throughput among all others. In addition, VFM has the second highest average throughput when compared to other simulations. Whereas, SM associated with 80 nodes has the lowest average throughput. Therefore, the simulation for various mobility speeds with the same number of nodes do not possess any large differences of average throughput between them. Except for the FM and VFM that are associated with 40 nodes have a big difference of average throughput when compared to the VSM, SM and MFM.

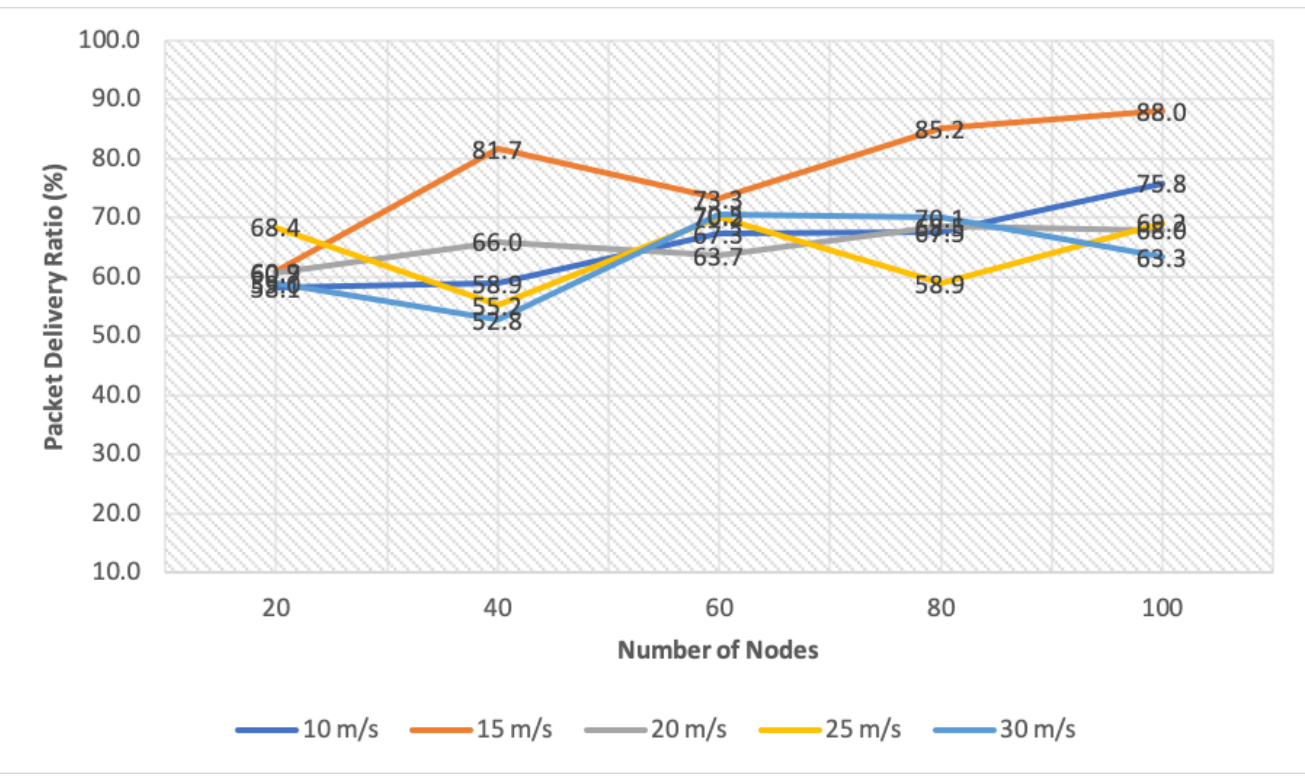

Figure 4. Packet delivery ratio. 
International Journal of Wireless \& Mobile Networks (IJWMN), Vol.13, No.6, December 2021

In Figure 4, SM has the higher percentage of PDR in all different numbers of nodes except for the number of nodes that are set to 20. This can be concluded that OLSR has a better packet delivery ratio when the mobility speed is set to SM when the number of nodes is set above 20. In addition, the SM with nodes being set to 100 has the highest packet delivery ratio. Whereas, the VFM with nodes being set to 40 has the lowest packet delivery ratio. The packet delivery ratio for various mobility speeds has slight differences when the number of nodes is set as 20 .

\section{Conclusions}

This paper explicates the application and result of OLSR performance by altering the speed of mobility and the network density. The network simulation for the OLSR protocol is executed and all the results obtained are recorded. From the result, the average delay graph illustrates an increasing trend as the number of nodes increases. While, both the packet delivery ratio graph and the average throughput graph showed not much of a change when the number of nodes increases. The mobility speed for different nodes does not show a huge difference with the same number of nodes given in simulation. The performance analysis and evaluation were done based on the result obtained. This is a crucial aspect of project development. Further study can be explored by implementing the approach in different scenario such as disaster area scenario with different type of mobility models and parameters for evaluating the network performance.

\section{CONFLICT OF INTEREST}

The authors declare no conflict of interest.

\section{ACKNOWLEDGMENTS}

This work was supported by the UniSZA/MENTORMENTEE/2018/022[R0046-R004] under the research of Performance Evaluation of Users Mobility Speed Over Mobile Cloud Computing.

\section{REFERENCES}

[1] S. Sivagurunathan and K. Prathapchandran, (2016) "Behaviour of Routing Protocols in Mobile Ad Hoc Networks Investigated for Emergency and Rescue Situation in India", International Journal of Wireless and Mobile Networks (IJWMN), Vol. 8, No. 1, pp. 23-37.

[2] N.A. Mahiddin, Fatin Fazain Mohd Affandi and Zarina Mohamad, (2021) "A review on mobility models in disaster area scenario", IJATEE, Vol. 8, No. 80, pp. 848-873.

[3] N. A. Mahiddin, N. I. Sarkar, and B. Cusack, (2017) "An Internet Access Solution: MANET Routing and a Gateway Selection Approach for Disaster Scenarios", The Review of Socionetwork Strategies.

[4] J. M. I. D. B. D. P. Hoebeke, (2004) "An overview of mobile ad hoc networks: Applications and challenges", JCN, Vol. 3, No. 3, pp. 60-66.

[5] N. A. Mahiddin and N. Sarkar, (2019) "An efficient gateway routing scheme for disaster recovery scenario".

[6] N. Aschenbruck and E. Gerhards-Padilla, (2008) "A survey on mobility models for performance analysis in tactical mobile networks", Journal of Telecommunications and Information Technology (ITL).

[7] Frikha, M., (2011) “Ad hoc networks: routing, QoS and optimization. London”, ISTE.

[8] Sarkar, S. K., Basavaraju, T. G., \& Puttamadappa, C., (2016) "Ad Hoc Mobile Wireless Networks Principles, Protocols, and Applications", Baton Rouge, in CRC Press.

[9] Ismail, R., Zulkifli, C. Z., \& Samsudin, K., (2016) "Routing Protocols for Mobile Ad-Hoc Network: A Qualitative Comparative Analysis", Jurnal Teknologi, Vol. 78, No. 8.

[10] Bai, Y., Mai, Y., \& Wang, N., (2017) "Performance comparison and evaluation of the proactive and reactive routing protocols for MANETs", Wireless Telecommunications Symposium (WTS). 
International Journal of Wireless \& Mobile Networks (IJWMN), Vol.13, No.6, December 2021

[11] Abdulleh, M. N. \& Yussof, S., (2015) "Performance Analysis of AODV, OLSR and GPSR MANET Routing Protocols with Respect to Network Size and Density", Research Journal of Applied Sciences, Engineering and Technology, Vol. 11, No. 4, pp. 400-406.

[12] Natarajan, K. \& Mahadevan, G., (2017) "Mobility based performance analysis of MANET routing protocols", IJCA, Vol. 163, No. 10, pp. 37-43.

[13] Lakshman Naik L, R. U. Khan and R. B. Mishra, (2016) "Analysis of Node Velocity Effects in MANET Routing Protocols using Network Simulator (NS3)”, IJCA, Vol. 144, No. 4, pp. 1-5.

[14] Gouri M. Patil, Ajay Kumar and A. D. Shaligram, (2016) "Performance Comparison of MANET Routing Protocols (OLSR, AODV, DSR, GRP and TORA) Considering Different Network Area Size", IJEMR, Vol. 6, No. 3, pp. 475-484.

[15] D. Kumar and S.C. Gupta, (2015) "Transmission Range, Density \& Speed based Performance Analysis of Ad Hoc Networks", African Journal of Computing \& ICT, Vol. 8, No. 1, pp. 173-178.

[16] Sharma, A., \& Kumar, R., (2016) "Performance comparison and detailed study of AODV, DSDV, DSR, TORA and OLSR routing protocols in ad hoc networks", 4th International Conference on Parallel, Distributed and Grid Computing (PDGC).

[17] Abdelkabir Sahnoun, Jamal El Abbadi and AhmedHabbani, (2017) "Multi-Metric Performance for OLSR Routing Protocol in Mobile Ad-Hoc Networks", International Journal of Wireless and Mobile Networks (IJWMN), Vol. 9, No. 3, pp. 39-49.

[18] R. H. R. Bongsu, A. Mohammed, and M. A. Mohamed, (2019) "Recent Trends in Channel Assignment Algorithms for Multi-Radio Multi-Channel in Wireless Mesh Network”, IJRTE, Vol. 7, No. $5 \mathrm{~S} 4$.

[19] Abdullah, A. M., Ozen, E., \& Bayramoglu, H., (2019) "Investigating the Impact of Mobility Models on MANET Routing Protocols", IJASCA, Vol. 10, No. 2.

[20] Moad, D., Djahel, S., \& Nait-Abdesselam, F., (2012) "Improving the quality of service routing in OLSR protocol," ICCIT.

\section{AUTHORS}

Koay Yong Cett received his Bachelor's degree in Computer Science of Network Security fromUniversiti Sultan Zainal Abidin, Terengganu, Malaysia in 2021. He was born in Kuala Lumpur on 5 February 1997. He is currently employed as a Cyber Security Engineer at LGMS - LE Global Services Sdn Bhd. His research interests include Network Security and Mobile Ad hoc Networks.

Nor Aida Mahiddin received the B.S. Degree in Information Technology from National University of Malaysia, the Master's Degree in Computer Science Major in Distributed Computing and Ph.D. degrees in Computer and Information Sciences from Auckland University of Technology, New Zealand. Dr. Nor Aida is currently a lecturer at Faculty of Informatics and Computing, University Sultan Zainal Abidin, Malaysia. She is the author of several papers in peer-reviewed journals and conferences proceeding. She is currently a member of the Institute of Electrical and Electronics Engineers (IEEE), Internet Society and The Society of Digital Information and Wireless Communications (SDIWC). Her research interests include network design, modelling and performance evaluation, wireless communication networks, disaster resilient network design, optimization of gateway congestion control, ad hoc and sensor networks and wireless mesh and routing protocols.

Fatin Fazain Mohd Affandi is a Computer Science's student, born and brought up in Besut, Terengganu, Malaysia. She did her Bachelors of Computer Science, major in Network Security in year 2019. Her area of interest is in Network and Security so, currently she is a PG student in the course named "Masters in Computer Science" at Universiti Sultan Zainal Abidin (UniSZA), Terengganu, Malaysia.
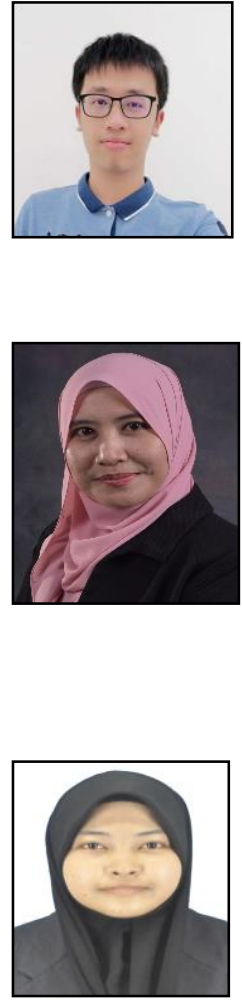
International Journal of Wireless \& Mobile Networks (IJWMN), Vol.13, No.6, December 2021

Raja Hasyifah Raja Bongsu received BS degree in Computer Science from University Kebangsaan Malaysia (UKM), in 2008 and MSc in Computer Science (Distributed Computing) from University Putra Malaysia (UPM), Malaysia. Her research interests are in the areas of distributed systems and computer networking, including wireless mesh networks.

Aznida Hayati Zakaria @ Mohamad hold Ph.D in Computer Science from University Malaysia Terengganu, M. Sc in Distributed Databases from the same university and a Bachelor's degree BIT majoring computer networking from University Utara Malaysia. Aznida Hayati works in University Sultan Zainal Abidin as faculty member and has several years work experience in the areas of teaching, research, administrative, programming, computer networking and arranging/organized research conferences, seminars, workshops, events. Dr. Aznida Hayati has several research publications in well-known international Journals and conferences.
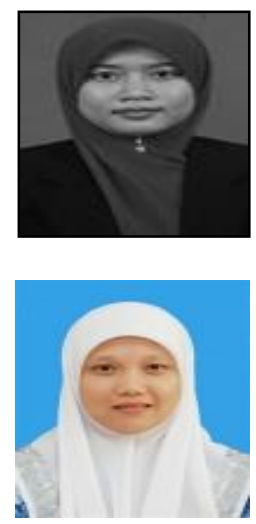